\title{
Helical Phase Inflation and Monodromy in Supergravity Theory
}

\author{
Tianjun Li, ${ }^{1,2}$ Zhijin Li, ${ }^{3}$ and Dimitri V. Nanopoulos ${ }^{3,4,5}$ \\ ${ }^{1}$ State Key Laboratory of Theoretical Physics and Kavli Institute for Theoretical Physics China (KITPC), Institute of Theoretical Physics, \\ Chinese Academy of Sciences, Beijing 100190, China \\ ${ }^{2}$ School of Physical Electronics, University of Electronic Science and Technology of China, Chengdu 610054, China \\ ${ }^{3}$ George P. and Cynthia W. Mitchell Institute for Fundamental Physics and Astronomy, Texas A\&M University, College Station, TX \\ 77843, USA \\ ${ }^{4}$ Astroparticle Physics Group, Houston Advanced Research Center (HARC), Mitchell Campus, The Woodlands, TX 77381, USA \\ ${ }^{5}$ Division of Natural Sciences, Academy of Athens, 28 Panepistimiou Avenue, 10679 Athens, Greece
}

Correspondence should be addressed to Zhijin Li; lizhijin@physics.tamu.edu

Received 3 July 2015; Accepted 17 November 2015

Academic Editor: Ignatios Antoniadis

Copyright (C) 2015 Tianjun Li et al. This is an open access article distributed under the Creative Commons Attribution License, which permits unrestricted use, distribution, and reproduction in any medium, provided the original work is properly cited. The publication of this article was funded by SCOAP ${ }^{3}$.

We study helical phase inflation which realizes "monodromy inflation" in supergravity theory. In the model, inflation is driven by the phase component of a complex field whose potential possesses helicoid structure. We construct phase monodromy based on explicitly breaking global $U(1)$ symmetry in the superpotential. By integrating out heavy fields, the phase monodromy from single complex scalar field is realized and the model fulfills natural inflation. The phase-axion alignment is achieved from explicitly symmetry breaking and gives super-Planckian phase decay constant. The $F$-term scalar potential provides strong field stabilization for all the scalars except inflaton, which is protected by the approximate global $U(1)$ symmetry. Besides, we show that helical phase inflation can be naturally realized in no-scale supergravity with $S U(2,1) / S U(2) \times U(1)$ symmetry since the supergravity setup needed for phase monodromy is automatically provided in the no-scale Kähler potential. We also demonstrate that helical phase inflation can be reduced to another well-known supergravity inflation model with shift symmetry. Helical phase inflation is free from the UV-sensitivity problem although there is super-Planckian field excursion, and it suggests that inflation can be effectively studied based on supersymmetric field theory while a UV-completed framework is not prerequisite.

\section{Introduction}

Inflation plays a crucial role in the early stage of our universe [1-3], and supersymmetry was found to be necessary for inflation soon after its discovery. A simple argument is that the inflation process is triggered close to the unification scale in Grand Unified Theory (GUT) $[4,5]$. At this scale physics theory is widely believed to be supersymmetric. To realize the slow-roll inflation, it requires strict flat conditions on the potential $V(\phi)$ of inflaton $\phi$. The mass of inflaton $m_{\phi}$ should be significantly smaller than the inflation energy scale due to the slow-roll parameter

$$
\eta \equiv M_{P}^{2} \frac{V^{\prime \prime}}{V} \simeq \frac{m_{\phi}^{2}}{3 H^{2}} \ll 1,
$$

where $M_{P}$ is the reduced Planck mass; otherwise, inflation cannot be triggered or last for a sufficient long period. However, as a scalar field, the inflaton is expected to obtain large quantum loop-corrections on the potential which can break the slow-roll conditions unless there is extremely fine tuning. Supersymmetry is a natural way to eliminate such quantum corrections. By introducing supersymmetry, the flatness problem can be partially relaxed but not completely solved since supersymmetry is broken during inflation. Moreover, gravity plays an important role in inflation, so it is natural to study inflation within supergravity theory.

Once combining the supersymmetry and gravity theory together, the flatness problem reappears known as $\eta$ problem. $N=1$ supergravity in four-dimensional space-time 
is determined by three functions: Kähler potential $K$, superpotential $W$, and gauge kinetic function. The $F$-term scalar potential contains an exponential factor $e^{K}$. In the minimal supergravity with $K=\Phi \bar{\Phi}$, the exponential factor $e^{K}$ introduces a term on the inflaton mass at Hubble scale, which breaks the slow-roll condition (1). To realize inflation in supergravity, the large contribution from $e^{K}$ to scalar mass should be suppressed, which needs a symmetry in $K$. In the minimal supergravity, $\eta$ problem can be solved by introducing shift symmetry in the Kähler potential as proposed by Kawasaki, Yamaguchi, and Yanagida (KYY) [6]: $K$ is invariant under the shift $\Phi \rightarrow \Phi+i C$. Consequently, $K$ is independent of $\operatorname{Im}(\Phi)$, so is the factor $e^{K}$ in the $F$ term potential. By employing $\operatorname{Im}(\Phi)$ as inflaton, its mass is not affected by $e^{K}$ and then there is no $\eta$ problem anymore. The shift symmetry can be slightly broken; in this case, there is still no $\eta$ problem and the model gives a broad range of tensor-to-scalar ratios $\mathbf{r}[7,8] . \eta$ problem is automatically solved in no-scale supergravity because of $S U(N, 1) / S U(N) \times$ $U(1)$ symmetry in the Kähler potential. Historically, the noscale supergravity was proposed to get vanishing cosmology constant. At classical level, the potential is strictly flat guaranteed by $S U(N, 1) / S U(N) \times U(1)$ symmetry of the Kähler potential, which meanwhile protects the no-scale type inflation away from $\eta$ problem. Moreover, $S U(N, 1) / S U(N) \times$ $U(1)$ symmetry has rich structure that allows different types of inflation. Thus, inflation based on no-scale supergravity has been extensively studied [9-19]. In this work, we will show that, in no-scale supergravity with $S U(2,1) / S U(2) \times U(1)$ symmetry, one can pick up $U(1)$ subsector, together with the superpotential phase monodromy to realize helical phase inflation.

Recently, it was shown that $\eta$ problem can be naturally solved in helical phase inflation [20]. This solution employs a global $U(1)$ symmetry, which is a trivial fact in the minimal supergravity with $K=\Phi \bar{\Phi}$. Using the phase of a complex field $\Phi$ as an inflaton, $\eta$ problem is solved due to the global $U(1)$ symmetry. The norm of $\Phi$ needs to be stabilized; otherwise, it will generate notable isocurvature perturbations that contradict the observations. However, it is a nontrivial task to stabilize the norm of $\Phi$ while keeping the phase light as the norm and phase couple with each other. In that work, the field stabilization and quadratic inflation are realized via a helicoid type potential. The inflationary trajectory is a helix line, and this is the reason for the name "helical phase inflation." In addition, the superpotential of helical phase inflation realizes monodromy in supersymmetric field theory. Furthermore, helical phase inflation gives a method to avoid the dangerous quantum gravity effect on inflation.

The single field slow-roll inflation agrees with recent observations $[4,5]$. Such kind of inflation admits a relationship between the inflaton field excursion and the tensor-toscalar ratio, which is known as the Lyth bound [21]. It suggests that, to get large tensor-to-scalar ratio, the field excursion during inflation should be much larger than the Planck mass. The super-Planckian field excursion challenges the validity, in the Wilsonian sense, of inflationary models described by effective field theory. At Planck scale, the quantum gravity effect is likely to introduce extra terms which are suppressed by the Planck mass and then irrelevant in the low energy scale, while for a super-Planckian field, the irrelevant terms become important and may introduce significant corrections or even destroy the inflation process. In this sense, the predictions just based on the effective field theory are not trustable. A more detailed discussion on the ultraviolet (UV) sensitivity of the inflation process is provided in [22].

A lot of works have been proposed to realize inflation based on the UV-completed theory, for example, in [2330]. However, to realize inflation in string theory, it needs to address several difficult problems such as moduli stabilization, Minkowski or de Sitter vacuum, and $\alpha^{\prime}$ - and higher string loop-corrections on the Kähler potential. However, one may doubt whether such difficult UV-completed framework is necessary for inflation. In certain scenario, the superPlanckian field excursion does not necessarily lead to the physical field above the Planck scale. A simple example is the phase of a complex field. The phase factor, like a pseudoNambu-Goldstone boson (PNGB), can be shifted to any value without any effect on the energy scale. By employing the phase as an inflaton, the super-Planckian field excursion is not problematic at all as there is no polynomial higher order quantum gravity correction for the phase component. Besides helical phase inflation, inflationary models using PNGB as an inflaton have been studied [31-39]. For natural inflation, it requires super-Planckian axion decay constant, which can be obtained by aligned axions [34] (the axion alignment relates to $S_{n}$ symmetry among Kähler moduli [40]) or anomalous $U(1)$ gauge symmetry with large condensation gauge group [41]. In helical phase inflation, as will be shown later, the phase monodromy in superpotential can be easily modified to generate natural inflation and also realize the super-Planckian phase decay constant, which is from the phase-axion alignment hidden in the process of integrating out heavy fields. Furthermore, all the extra fields are consistently stabilized based on the helicoid potential.

Like helical phase inflation, "monodromy inflation" was proposed to solve the UV sensitivity of large field inflation $[42,43]$. In such model, the inflaton is identified as an axion obtained from $p$-form field after string compactifications. The inflaton potential arises from the DBI action of branes or coupling between axion and fluxes. During inflation, the axion rotates along internal cycles and reduces the axion potential slowly, while all the other physical parameters are unaffected by the axion rotation. Interesting realization of monodromy inflation is the axion alignment [34], which was proposed to get super-Planckian axion decay constant for natural inflation, and it was noticed that this mechanism actually provides an axion monodromy in [44-46]. Actually, a similar name "helical inflation" was firstly introduced in [45] for an inflation model with axion monodromy. However, a major difference should be noted; the "helical" structure in [45] is to describe the alignment structure of two axions, while the "helical" structure in our model is from a single complex field with stabilized field norm. The physical picture of axion monodromy is analogical to the superpotential $W$ in 
helical phase inflation. For $W$, there is monodromy around the singularity $\Phi=0$ :

$$
\begin{gathered}
\Phi \longrightarrow \Phi e^{2 \pi i}, \\
W \longrightarrow W+2 \pi i \frac{W}{\log \Phi} .
\end{gathered}
$$

The phase monodromy, together with $U(1)$ symmetry in the Kähler potential, provides flat direction for inflation. In the following, we will show that this monodromy is corresponding to the global $U(1)$ symmetry explicitly broken by the inflation term.

In this work, we will study helical phase inflation from several aspects in detail. Firstly, we will show that the phase monodromy in the superpotential, which leads to the helicoid structure of inflaton potential, can be effectively generated by integrating out heavy fields in supersymmetric field theory. Besides quadratic inflation, the phase monodromy for helical phase inflation can be easily modified to realize natural inflation, in which the process of integrating out heavy fields fulfills the phase-axion alignment indirectly and leads to super-Planckian phase decay constant with consistent field stabilization as well. We also show that helical phase inflation can be reduced to the KYY inflation by field redefinition; however, there is no such field transformation that can map the KYY model back to helical phase inflation. Furthermore, we show that the no-scale supergravity with $S U(2,1) / S U(2) \times U(1)$ symmetry provides a natural frame for helical phase inflation, as $S U(2,1) / S U(2) \times U(1)$ symmetry of no-scale Kähler potential already combines the symmetry factors needed for phase monodromy. Moreover, we argue that helical phase inflation is free from the UV-sensitivity problem.

This paper is organized as follows. In Section 2, we review the minimal supergravity construction of helical phase inflation. In Section 3, we present the realization of phase monodromy based on supersymmetric field theory. In Section 4, natural inflation as a special type of helical phase inflation is studied. In Section 5, the relationship between helical phase inflation and the KYY model is discussed. In Section 6, we study helical phase inflation in no-scale supergravity with $S U(2,1) / S U(2) \times U(1)$ symmetry. In Section 7 , we discuss how helical phase inflation dodges the UV-sensitivity problem of large field inflation. Conclusion is given in Section 8.

\section{Helical Phase Inflation}

In four dimensions, $N=1$ supergravity is determined by the Kähler potential $K$, superpotential $W$, and gauge kinetic function. The $F$-term scalar potential is given by

$$
V=e^{K}\left(K^{i \bar{j}} D_{i} W D_{\bar{j}} \bar{W}-3 W \bar{W}\right) .
$$

To realize inflation in supergravity, the factor $e^{K}$ in the above formula is an obstacle as it makes the potential too steep for a sufficient long slow-roll process. This is the well-known $\eta$ problem. To solve $\eta$ problem, usually one needs a symmetry in the Kähler potential. In the minimal supergravity, there is a global $U(1)$ symmetry in the Kähler potential $K=\Phi \bar{\Phi}$. This global $U(1)$ symmetry is employed in helical phase inflation. As the Kähler potential is independent of the phase $\theta$, the potential of phase $\theta$ is not affected by the exponential factor $e^{K}$. Consequently, there is no $\eta$ problem for phase inflation. However, the field stabilization becomes more subtle. All the extra fields except inflaton have to be stabilized for single field inflation, but normally the phase and norm of a complex field couple with each other and then it is very difficult to stabilize norm while keeping phase light.

The physical picture of helical phase inflation is that the phase evolves along a flat circular path with constant, or almost constant, radius-the field magnitude, and the potential decreases slowly. So even before writing down the explicit supergravity formula, one can deduce that phase inflation, if realizable, should be particular realization of complex phase monodromy, and there exists a singularity in the superpotential that generates the phase monodromy. Such singularity further indicates that the model is described by an effective theory.

Helical phase inflation is realized in the minimal supergravity with the Kähler potential

$$
K=\Phi \bar{\Phi}+X \bar{X}-g(X \bar{X})^{2}
$$

and superpotential

$$
W=a \frac{X}{\Phi} \ln (\Phi) .
$$

The global $U(1)$ symmetry in $K$ is broken by the superpotential with a small factor $a$; when $a \rightarrow 0, U(1)$ symmetry is restored. Therefore, the superpotential with small coefficient is technically natural [47], which makes the model technically stable against radiative corrections. As discussed before, the superpotential $W$ is singular at $\Phi=0$ and exhibits a phase monodromy

$$
\begin{gathered}
\Phi \longrightarrow \Phi e^{2 \pi i}, \\
W \longrightarrow W+2 \pi a i \frac{X}{\Phi} .
\end{gathered}
$$

The theory is well defined only for $\Phi$ away from the singularity.

During inflation, the field $X$ is stabilized at $X=0$, and the scalar potential is simplified as

$$
V=e^{\Phi \bar{\Phi}} W_{X} \bar{W}_{\bar{X}}=a^{2} e^{r^{2}} \frac{1}{r^{2}}\left((\ln r)^{2}+\theta^{2}\right),
$$

where $\Phi=r e^{i \theta}$, and the kinetic term is $L_{K}=\partial_{\mu} r \partial^{\mu} r+$ $r^{2} \partial_{\mu} \theta \partial^{\mu} \theta$. Interestingly, in the potential (7), both the normdependent factor $e^{r^{2}}\left(1 / r^{2}\right)$ and $(\ln r)^{2}$ reach the minimum at $r=1$. The physical mass of norm $r$ is

$$
m_{r}^{2}=\left.\frac{1}{2} \frac{\partial^{2} V}{\partial r^{2}}\right|_{r=1}=\left(2+\frac{1}{\theta^{2}}\right) V_{I}
$$


therefore, the norm is strongly stabilized at $r=1$ during inflation and the Lagrangian for the inflaton is

$$
L=\partial_{\mu} \theta \partial^{\mu} \theta-e a^{2} \theta^{2},
$$

which gives quadratic inflation driven by the phase of complex field $\Phi$.

In the above simple example given by (4) and (5), the field stabilization is obtained from the combination of supergravity correction $e^{K}$ and the pole $1 / \Phi$ in $W$, besides an accidental agreement that both the factor $\left(1 / r^{2}\right) e^{r^{2}}$ and the term $(\ln r)^{2}$ obtain their minima at $r=1$, while for more general helical phase inflation, such accidental agreement is not guaranteed. For example, one may get the following inflaton potential:

$$
V=e^{K(r)} \frac{1}{r^{2}}\left(\left(\ln \frac{r}{\Lambda}\right)^{2}+\theta^{2}\right),
$$

in which the coefficient $e^{K(r)}\left(1 / r^{2}\right)$ admits a minimum at $r_{0} \sim \Lambda$ but $r_{0} \neq \Lambda$. In this case, the coefficient $e^{K(r)}\left(1 / r^{2}\right)$ still gives a mass above the Hubble scale for $r$, while $\langle r\rangle$ is slightly shifted away from $r_{0}$ in the early stage of inflation, and after inflation $r$ evolves to $\Lambda$ rapidly. Also, the term $(\ln (r / \Lambda))^{2}$ gives a small correction to the potential and inflationary observables, so this correction is ignorable comparing with the contributions from the super-Planckian valued phase unless it is unexpectedly large.

Potential Deformations. In the Kähler potential, there are corrections from the quantum loop effect, while the superpotential $W$ is nonrenormalized. Besides, when coupled with heavy fields, the Kähler potential of $\Phi$ receives corrections through integrating out the heavy fields. Nevertheless, because of the global $U(1)$ symmetry in the Kähler potential, these corrections can only affect the field stabilization, while phase inflation is not sensitive to these corrections.

Given a higher order correction on the Kähler potential

$$
K=\Phi \bar{\Phi}+b(\Phi \bar{\Phi})^{2}+X \bar{X}-g(X \bar{X})^{2},
$$

one may introduce an extra parameter $\Lambda$ in the superpotential

$$
W=a \frac{X}{\Phi} \ln \frac{\Phi}{\Lambda} .
$$

Based on the same argument, it is easy to see the scalar potential reduces to

$$
V=a^{2} e^{r^{2}+b r^{4}} \frac{1}{r^{2}}\left((\ln r-\ln \Lambda)^{2}+\theta^{2}\right) .
$$

The factor $e^{r^{2}+b r^{4}}\left(1 / r^{2}\right)$ reaches its minimum at $r_{0}^{2}=2 /(1+$ $\sqrt{1+8 b}$ ), below $M_{P}$ for $b>0$. To get the "accidental agreement" it needs the parameter $\Lambda=r_{0}$, and then inflation is still driven by the phase with exact quadratic potential.

Without $\Lambda$, the superpotential comes back to (5) and the scalar potential is shown in Figure 1 with $b=0.1$. During inflation $\langle r\rangle \simeq r_{0}$ for small $b$, the term $(\ln r)^{2}$ contribution to

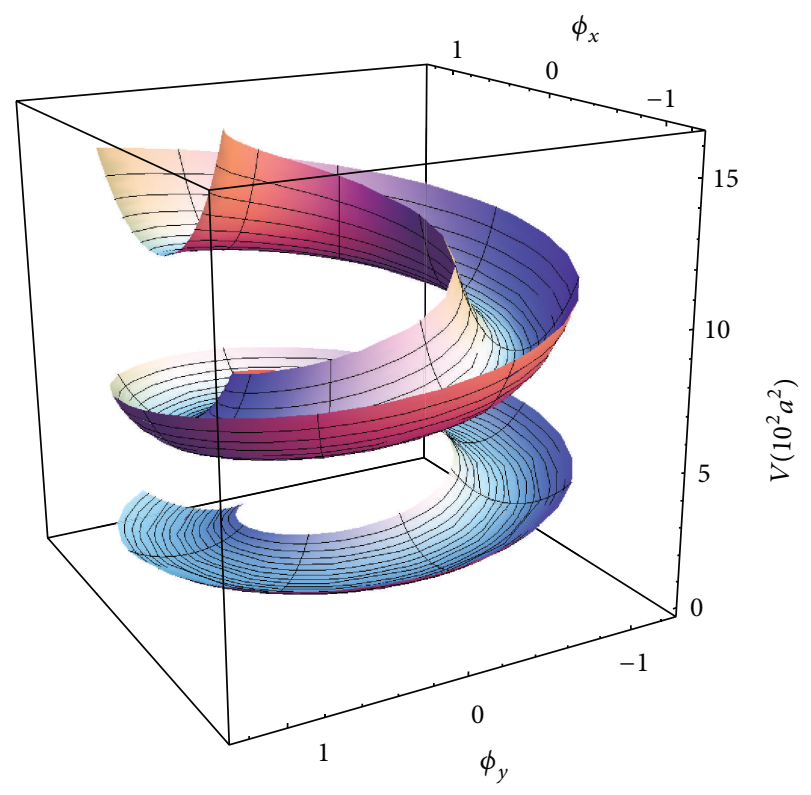

FIGURE 1: The helicoid structure of potential (13) scaled by $10^{2} a^{2}$. In the graph, parameters $b$ and $\Lambda$ are set to be $b=0.1$ and $\Lambda=1$, respectively.

the potential, at the lowest order, is proportional to $b^{2}$. After canonical field normalization, the inflaton potential takes the form

$$
V(\theta)=\frac{1}{2} m_{\theta}^{2}\left(2 b^{2}+\theta^{2}\right),
$$

in which the higher order terms proportional to $b^{3+i}$ are ignored. With regard to the inflationary observations, take the tensor-to-scalar ratio $\mathbf{r}$, for example, as

$$
\mathbf{r}=\frac{32 \theta_{i}^{2}}{\left(\theta_{i}^{2}+2 b^{2}\right)^{2}} \approx \frac{8}{N}\left(1+\frac{b^{2}}{2 N}\right)^{-2},
$$

where $\theta_{i}$ is the phase when inflation starts and $N \in(50,60)$ is the $e$-folding number. So the correction from higher order term is insignificant for $b<1$.

\section{Monodromy in Supersymmetric Field Theory}

As discussed before, phase inflation naturally leads to the phase monodromy (in mathematical sense) in the superpotential. The phase monodromy requires singularity, which means the superpotential proposed for phase inflation should be an effective theory. It is preferred to show how such phase monodromy appears from a more "fundamental" theory at higher scale. In [20], the monodromy needed for phase inflation is realized based on the supersymmetric field theory, in which the monodromy relates to the soft breaking of a global $U(1)$ symmetry.

Historically, the monodromy inflation as an attractive method to realize super-Planckian field excursion was first proposed, in a more physical sense, for axions arising from 
string compactifications [42]. In the inflaton potential, the only factor that changes during axion circular rotation is from the DBI action of branes. In [44-46], the generalized axion alignment mechanisms are considered as particular realization of axion monodromy with the potential from nonperturbative effects. We will show that such kind of axion monodromy can also be fulfilled by the superpotential phase monodromy [20], even though it is not shown in the effective superpotential after integrating out the heavy fields. Furthermore, all the extra fields can be consistently stabilized.

The more "fundamental" field theory for the superpotential in (5) is

$$
W_{0}=\sigma X \Psi(T-\delta)+Y\left(e^{-\alpha T}-\beta \Psi\right)+Z(\Psi \Phi-\lambda),
$$

where the coupling constants for the second and third terms are taken to be 1 for simplicity, and a small hierarchy is assumed between the first term and the last two terms; that is, $\sigma \ll 1$. The coupling $Y e^{-\alpha T}$ is assumed to be an effective description of certain nonperturbative effects. Similar forms can be obtained from $D$-brane instanton effect in type string theory (for a review, see [48]), besides the coefficient $\alpha \propto$ $1 / f \gg 1$ in Planck unit, since $f \ll 1$ is the decay constant and should be significantly lower than the Planck scale. For the last two terms of $W_{0}$, there is a global $U(1)$ symmetry:

$$
\begin{aligned}
& \Psi \longrightarrow \Psi e^{-i q \theta}, \\
& \Phi \longrightarrow \Phi e^{i q \theta}, \\
& Y \longrightarrow Y e^{i q \theta}, \\
& T \longrightarrow T+\frac{i q \theta}{\alpha},
\end{aligned}
$$

which is anomalous and explicitly broken by the first term. Phase rotation of the stabilizer field $X$ has no effect on inflation and so is ignored here. The phase monodromy of superpotential $W$ in (6) originates from the $U(1)$ rotation of $W_{0}$ :

$$
\begin{gathered}
\Psi \longrightarrow \Psi e^{-i 2 \pi}, \\
W_{0} \longrightarrow W_{0}+i 2 \pi \sigma \frac{1}{\alpha} X \Psi .
\end{gathered}
$$

As shown in [20], the supersymmetric field theory with superpotential $W_{0}$ admits the Minkowski vacuum at

$$
\begin{aligned}
& \langle X\rangle=\langle Y\rangle=\langle Z\rangle=0, \\
& \langle T\rangle=\delta \\
& \langle\Psi\rangle=\frac{1}{\beta} e^{-\alpha \delta}, \\
& \langle\Phi\rangle=\lambda \beta e^{\alpha \delta},
\end{aligned}
$$

with $\langle\Phi\rangle \gg\langle\Psi\rangle$ so that near the vacuum the masses of $Y$, $Z$, and $\Psi$ are much larger than $\Phi$; besides, the effective mass of $T$ is also large near the vacuum due to large $\alpha$. Large $\alpha$ was an obstacle for natural inflation as it leads to the axion decays too small for inflation, while in this scenario large $\alpha$ is helpful for phase inflation to stabilize the axion. Therefore, for the physical process at scale below the mass scale of three heavy fields, the only unfixed degrees of freedom are $X$ and $\Phi$, which can be described by an effective field theory with three heavy fields integrated out. The coupling $\sigma X \Psi(T-\delta)$ is designed for inflation and hierarchically smaller than the extra terms in $W_{0}$. Therefore, to describe inflation process, the heavy fields need to be integrated out.

To integrate out heavy fields, we need to consider the $F$ terms again. The $F$-term flatness of fields $Y$ and $Z$ gives

$$
\begin{aligned}
& F_{Y}=e^{-\alpha T}-\beta \Psi+K_{Y} W_{0}=0, \\
& F_{Z}=\Psi \Phi-\lambda+K_{Z} W_{0}=0 .
\end{aligned}
$$

Near the vacuum $Y=Z \approx 0 \ll M_{P}$, the above supergravity corrections $K_{Y(Z)} W_{0}$ are ignorable, and then the $F$-term flatness conditions reduce to these for global supersymmetry. This is gained from the fact that although the inflation dynamics are subtle, the inflation energy density is close to the GUT scale, far below the Planck scale. Solving the $F$-term flatness equations in (20), we obtain the effective superpotential $W$ in (5).

Based on the above construction, it is clear that the phase monodromy in $W$ is from the $U(1)$ transformation of $W_{0}$, and the pole of superpotential (5) at $\Phi=0$ arises from the integration process. The heavy field $\Psi$ is integrated out based on the $F$-term flatness conditions when $\langle\Phi\rangle \gg\langle\Psi\rangle$, while if $\Phi \rightarrow 0, \Psi$ becomes massless from $\left|F_{Z}\right|^{2}$ and it is illegal to integrate out a "massless" field. For inflation, the condition $\langle\Phi\rangle \gg\langle\Psi\rangle$ is satisfied so the theory with superpotential (5) is reliable.

As to the inflation term, a question appears: as global $U(1)$ is explicitly broken by the first term in (16) at inflation scale, why is the phase light while the norm is much heavier? The supergravity correction to the scalar potential plays a crucial role at this stage. The coefficient $e^{K}$ appears in the scalar potential, and because of $U(1)$ symmetry in the Kähler potential, the factor $e^{K}$ is invariant under $U(1)$ symmetry but increases exponentially for a large norm. Here, the Kähler potential of $T$ should be shift invariant; that is, $K=K(T+$ $\bar{T})$ instead of the minimal type. Otherwise, the exponential factor $e^{K}$ depends on phase as well and the phase rotation will be strongly fixed, like the norm component or $\operatorname{Re}(T)$.

When integrating out the heavy fields, they should be replaced both in superpotential and in Kähler potential by the solutions from vanishing $F$-term equations. So, different from the superpotential, the Kähler potential obtained in this way is slightly different from the minimal case given in (4). There are extra terms like

$$
\begin{gathered}
\Psi \bar{\Psi}=\frac{\lambda^{2}}{r^{2}}, \\
K(T+\bar{T})=K\left(\frac{1}{\alpha^{2}}(\ln r)^{2}\right),
\end{gathered}
$$

where $|\Phi|=r$. Nevertheless, since $\lambda \ll 1$ and $\alpha \gg 1$, these terms are rather small and have little effect on phase inflation, 
as shown in the last section. Furthermore, the quantum loop effects during integrating out heavy fields can introduce corrections to the Kähler potential as well. However, because of $U(1)$ symmetry built in the Kähler potential, these terms, together with (21), can only mildly affect the field stabilization, and phase inflation is not sensitive to the corrections in Kähler potential. As to the superpotential, it is protected by the nonrenormalized theorem and free from radiative corrections.

\section{Natural Inflation in Helical Phase Inflation}

In the superpotential $W_{0}$, the inflation term is perturbative coupling of complex field $T$ which shifts under global $U(1)$; an interesting modification is to consider inflation given by nonperturbative coupling of $T$. Such term gives a modified $U(1)$ phase monodromy in the superpotential. And it leads to natural inflation as a special type of helical phase inflation with phase-axion alignment, which is similar to the axion-axion alignment mechanism proposed in [34] for natural inflation with super-Planckian axion decay constant. Specifically, it can be shown that the phase monodromy realized in supersymmetric field theory has similar physical picture to the modified axion alignment mechanism provided in $[44,45]$.

To realize natural inflation, the superpotential $W_{0}$ in (16) just needs to be slightly modified:

$$
\begin{aligned}
W_{1}= & \sigma X \Psi\left(e^{-\alpha T}-\delta\right)+Y\left(e^{-\beta T}-\mu \Psi\right) \\
& +Z(\Psi \Phi-\lambda)
\end{aligned}
$$

in which $1 \ll \alpha \ll \beta$. Again there is a global $U(1)$ symmetry in the last two terms of $W_{1}$, and the fields transfer under $U(1)$ like in (17). The first term, which is hierarchically smaller, breaks the $U(1)$ symmetry explicitly; besides, a shift symmetry of $T$ is needed in the Kähler potential. The monodromy of $W_{1}$ under a circular $U(1)$ rotation is

$$
\begin{aligned}
\Psi & \longrightarrow \Psi e^{-i 2 \pi}, \\
W_{1} & \longrightarrow W_{1}+\sigma X \Psi e^{-\alpha T}\left(e^{-2 \pi i(\alpha / \beta)}-1\right) .
\end{aligned}
$$

The supersymmetric field theory given by (22) admits the following supersymmetric Minkowski vacuum:

$$
\begin{aligned}
& \langle X\rangle=\langle Y\rangle=\langle Z\rangle=0, \\
& \langle T\rangle=-\frac{1}{\alpha} \ln \delta, \\
& \langle\Psi\rangle=\frac{1}{\mu} \delta^{\beta / \alpha} \\
& \langle\Phi\rangle=\lambda \mu \delta^{-\beta / \alpha}
\end{aligned}
$$

The parameters are set to satisfy conditions

$$
\begin{aligned}
\delta^{\beta / \alpha} & \sim \lambda \mu, \\
\lambda \ll 1, & \ll<1,
\end{aligned}
$$

so that $\langle\Psi\rangle \ll\langle\Phi\rangle$ and $\langle T\rangle>0$.
Near vacuum, fields $Y, Z, \Psi$, and $T$ obtain large effective masses above inflation scale while $X, \Phi$ are much lighter. At the inflation scale, the heavy fields should be integrated out. The $F$-term flatness conditions for fields $Y$ and $Z$ are

$$
\begin{aligned}
& F_{Y}=e^{-\beta T}-\mu \Psi=0, \\
& F_{Z}=\Psi \Phi-\lambda=0,
\end{aligned}
$$

in which the supergravity corrections $K_{Y / Z} W_{1}$ are neglected as both $Y$ and $Z$ get close to zero during inflation. Integrating out $\Psi$ and $T$ from (26), we obtain the effective superpotential $W^{\prime}$ from $W_{1}$ :

$$
W^{\prime}=\sigma \lambda \frac{X}{\Phi}\left(\left(\frac{\mu \lambda}{\Phi}\right)^{\alpha / \beta}-\delta\right)
$$

Given $\alpha \ll \beta$, the effective superpotential contains a term with fractional power. Inflation driven by complex potential with fractional power was considered in [49] to get sufficient large axion decay constant. Here, the supersymmetric field monodromy naturally leads to the superpotential with fractional power, which arises from the small hierarchy of axion decay constants in two nonperturbative terms.

Helical phase inflation is described by the effective superpotential $W^{\prime}$; the role of phase-axion alignment is not clear from $W^{\prime}$ since it is hidden in the procedure of integrating out heavy fields.

The F-term flatness conditions (26) fix four degrees of freedom; for the extra degree of freedom, they correspond to the transformations free from constraints (26):

$$
\begin{aligned}
& \Psi \longrightarrow \Psi e^{-u-i v}, \\
& \Phi \longrightarrow \Phi e^{u+i v}, \\
& T \longrightarrow T+\frac{u}{\beta}+\frac{i v}{\beta} .
\end{aligned}
$$

Parameter $u$ corresponds to the norm variation of complex field $\Phi$, which is fixed by the supergravity correction on the scalar potential $e^{K(\Phi \bar{\Phi})}$. Parameter $v$ relates to $U(1)$ transformation, which leads to the phase monodromy from the first term of $W_{1}$. The scalar potential, including the inflation term, depends on the superpositions among phases of $\Psi, \Phi$ and the axion $\operatorname{Im}(T)$, which are constrained as in (28). Among these fields, the phase of $\Phi$ has the lightest mass after canonical field normalization. Similar physical picture appears in the axionaxion alignment where inflation is triggered by the axion superposition along the flat direction.

After integrating out the heavy fields, the Kähler potential is

$$
K=\Phi \bar{\Phi}+\frac{\lambda^{2}}{\Phi \bar{\Phi}}+\cdots
$$

As $\lambda \ll 1$, the Kähler potential is dominated by $\Phi \bar{\Phi}$; as discussed before, the extra terms like $\lambda^{2} / \Phi \bar{\Phi}$ only give small 
corrections to the field stabilization. Helical phase inflation can be simply described by the supergravity

$$
\begin{aligned}
& K=\Phi \bar{\Phi}+X \bar{X}+\ldots, \\
& W=a \frac{X}{\Phi}\left(\Phi^{-b}-c\right),
\end{aligned}
$$

where $b=\alpha / \beta \ll 1$ and $c \approx 1$. The scalar potential given by the above Kähler potential and superpotential is

$$
V=e^{r^{2}} \frac{a^{2}}{r^{2}}\left(r^{-2 b}+c^{2}-2 c r^{-b} \cos (b \theta)\right),
$$

in which we have used $\Phi \equiv r e^{i \theta}$. As usual, the norm $r$ couples with the phase in the scalar potential. To generate single field inflation, the norm component needs to be stabilized with heavy mass above the Hubble scale while the phase component remains light. Due to the phase-norm coupling, the phase and norm are likely to obtain comparable effective masses during inflation so it is difficult to realize single field inflation. However, it is a bit different in helical phase inflation. The above scalar potential can be rewritten as follows:

$$
V=e^{r^{2}} \frac{a^{2}}{r^{2}}\left(r^{-b}-c\right)^{2}+e^{r^{2}} \frac{4 a^{2} c}{r^{2+b}}\left(\sin \frac{b}{2} \theta\right)^{2}
$$

So its vacuum locates at $\langle r\rangle=r_{0}=c^{-1 / b}$ and $\theta=0$. Besides, the coefficient of the phase term $e^{r^{2}}\left(4 c / r^{2+b}\right)$ reaches its minimal value at $r_{1}=\sqrt{1+b / 2}$. For $c \approx 1$, we have the approximation $r_{0} \approx r_{1} \approx 1$. The extra terms in $K$ give small corrections to $r_{0}$ and $r_{1}$, but the approximation is still valid. So with the parameters in (25), the norm $r$ in the two terms of scalar potential $V$ can be stabilized at the close region $r \approx 1$ separately. If the parameters are tuned so that $r_{0}=r_{1}$, then the norm of complex field is strictly stabilized at the vacuum value during inflation. Without such tuning, a small difference between $r_{0}$ and $r_{1}$ is expected but the shift of $r$ during inflation is rather small and inflation is still approximate to the single field inflation driven by the phase term $\propto(\sin (b / 2) \theta)^{2}$. The helicoid structure of the potential (31) is shown in Figure 2.

It is known that, to realize aligned axion mechanism in supergravity [50], it is very difficult to stabilize the moduli as they couple with the axions. In [40], the moduli stabilization is fulfilled with gauged anomalous $U(1)$ symmetries, since $U(1) D$-terms only depend on the norm $|\Phi|$ or $\operatorname{Re}(T)$ and then directly separate the norms and phases of matter fields. In helical phase inflation, the modulus and matter fields except the phase are stabilized at higher scale or by the supergravity scalar potential. Only the phase can be an inflaton candidate because of the protection from the global $U(1)$ symmetry in Kähler potential and approximate $U(1)$ symmetry in superpotential.

\section{Helical Phase Inflation and the KYY Model}

$\eta$ problem in supergravity inflation can be solved both in helical phase inflation and in the shift symmetry in KYY

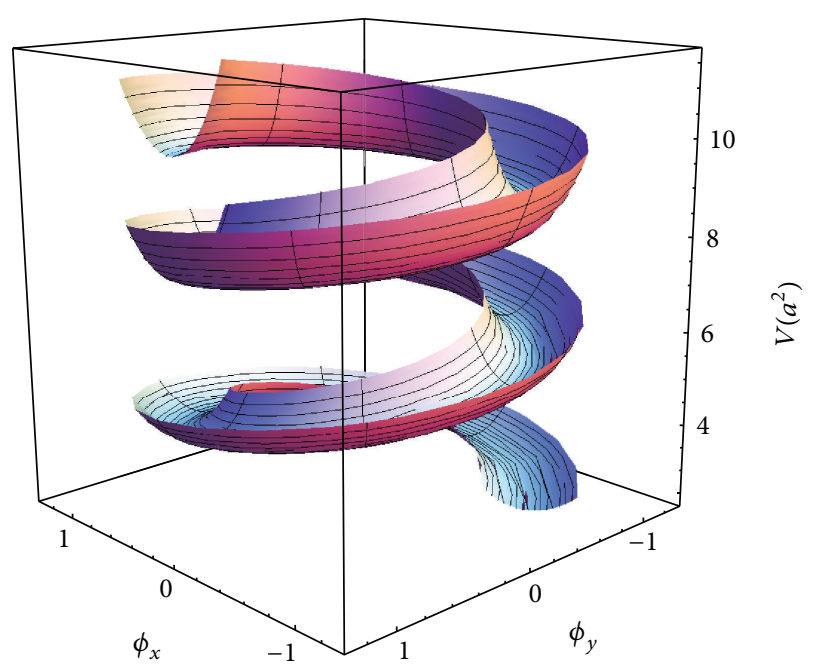

Figure 2: The helicoid structure of potential (31) scaled by $a^{2}$. The parameters with $c=0.96$ and $b=0.1$ are adopted in the graph. It is shown that the local valley locates around $r \approx 1$. Note that the potential gets flatter at the top of the graph.

model. The physics in these two solutions are obviously different. For helical phase inflation, the solution employs $U(1)$ symmetry in Kähler potential of the minimal supergravity, and the superpotential admits a phase monodromy arising from the global $U(1)$ transformation:

$$
\begin{aligned}
K & =\Phi \bar{\Phi}+X \bar{X}+\cdots, \\
W & =a \frac{X}{\Phi} \ln \Phi .
\end{aligned}
$$

The inflation is driven by the phase of complex field $\Phi$ and several special virtues appear in the model. For the KYY model with shift symmetry [6], the Kähler potential is adjusted so that it admits a shift symmetry along the direction of $\operatorname{Im}(T)$ :

$$
\begin{aligned}
K & =\frac{1}{2}(T+\bar{T})^{2}+X \bar{X}+\cdots, \\
W & =a X T,
\end{aligned}
$$

and inflation is driven by $\operatorname{Im}(T)$. The shift symmetry is endowed with axions so this mechanism is attractive for axion inflation.

Here, we will show that although the physical pictures are much different in helical phase inflation and KYY type model, just considering the lower order terms in the Kähler potential of redefined complex field, helical phase inflation can reduce to the KYY model.

Because the phase of $\Phi$ rotates under the global $U(1)$ transformation, to connect helical phase inflation with the KYY model, a natural guess is to take the following field redefinition $\Phi=e^{T}$; then helical phase inflation (33) becomes

$$
\begin{aligned}
& K=e^{T+\bar{T}}+\cdots=1+T+\bar{T}+\frac{1}{2}(T+\bar{T})^{2}+\cdots, \\
& W=a X T e^{-T} .
\end{aligned}
$$


The Kähler manifold of complex field $T$ is invariant under the holomorphic Kähler transformation

$$
K(T, \bar{T}) \longrightarrow K(T, \bar{T})+F(T)+\bar{F}(\bar{T})
$$

in which $F(T)$ is a holomorphic function of $T$. To keep the whole Lagrangian also invariant under the Kähler transformation, the superpotential transforms under the Kähler transformation

$$
W \longrightarrow e^{-F(T)} W
$$

For the supergravity model in (35), taking the Kähler transformation with $F(T)=-(1 / 2)-T$, the Kähler potential and superpotential become

$$
\begin{aligned}
K & =\frac{1}{2}(T+\bar{T})^{2}+\cdots, \\
W & =a \sqrt{e} X T
\end{aligned}
$$

which is just the KYY model (34) with higher order corrections in the Kähler potential. These higher order terms vanish after field stabilization and have no effect on inflation process. The field relation $\Phi=e^{T}$ also gives a map between the simplest helical phase inflation and the KYY model, such as the inflaton, $\arg (\Phi) \rightarrow \operatorname{Im}(T)$, and field stabilization $|\Phi|=1 \rightarrow \operatorname{Re}(T)=0$.

Nevertheless, helical phase inflation is not equivalent to the KYY model. There are higher order corrections to the Kähler potential in the map from helical phase inflation to KYY model, which have no effect on inflation after field stabilization but indicate different physics in two models. By dropping these terms, certain information is lost so the map is irreversible. Specifically, the inverse function $T=\ln \Phi$ of the field redefinition $\Phi=e^{T}$ cannot reproduce helical phase inflation from the KYY model, unless one introduces higher dimensional operators in the Kähler potential exactly the same as given in the expansion (35). Such strict constraint on each higher dimensional operator is not guaranteed by any known rule and so is unphysical. As there is no pole or singularity in the KYY model, it is unlikely to introduce pole and singularity at origin with phase monodromy through welldefined field redefinition. Actually, the singularity with phase monodromy in the superpotential indicates rich physics in the scale above inflation.

\section{Helical Phase Inflation in No-Scale Supergravity}

The no-scale supergravity is an attractive frame for GUT scale phenomenology; it is interesting to realize helical phase inflation in no-scale supergravity. Generally, the Kähler manifold of the no-scale supergravity is equipped with $S U(N, 1) / S U(N) \times U(1)$ symmetry. For the no-scale supergravity with exact $S U(1,1) / U(1)$ symmetry, without extra fields no inflation can be realized, so the case with $S U(2,1) / S U(2) \times U(1)$ symmetry is the simplest one that admits inflation.
The Kähler potential with $S U(2,1) / S U(2) \times U(1)$ symmetry is

$$
K=-3 \ln \left(T+\bar{T}-\frac{\Phi \bar{\Phi}}{3}\right)
$$

In the symmetry of the Kähler manifold, there is $U(1)$ subsector, the phase rotation of complex field $\Phi$, which can be employed for helical phase inflation. Besides, the modulus $T$ should be stabilized during inflation, which can be fulfilled by introducing extra terms on $T$. As a simple example of no-scale helical phase inflation, here we follow the simplification in [9] that the modulus $T$ has already been stabilized at $\langle T\rangle=c$. Different from the minimal supergravity, the kinetic term given by the no-scale Kähler potential is noncanonical:

$$
\begin{aligned}
L_{K} & =K_{\Phi \Phi} \partial_{\mu} \Phi \partial^{\mu} \bar{\Phi} \\
& =\frac{2 c}{\left(2 c-r^{2} / 3\right)^{2}}\left(\partial_{\mu} r \partial^{\mu} r+r^{2} \partial_{\mu} \theta \partial^{\mu} \theta\right)
\end{aligned}
$$

in which $\Phi \equiv r e^{i \theta}$ is used. The $F$-term scalar potential is

$$
V=e^{-(2 / 3) K}\left|W_{\Phi}\right|^{2}=\frac{\left|W_{\Phi}\right|^{2}}{(T+\bar{T}-\Phi \bar{\Phi} / 3)^{2}},
$$

where the superpotential $W$ is a holomorphic function of superfield $\Phi$ and $T=\langle T\rangle=c$. It requires a phase monodromy in superpotential $W$ for phase inflation; the simple choice is

$$
W=\frac{a}{\Phi} \ln \frac{\Phi}{\Lambda} .
$$

The scalar potential given by this superpotential is

$$
V=\frac{9 a^{2}}{\left(6 c-r^{2}\right)^{2} r^{4}}\left(\left(\ln \frac{r}{e \Lambda}\right)^{2}+\theta^{2}\right) .
$$

As in the minimal supergravity, the norm and phase of complex field $\Phi$ are separated in the scalar potential. For the $r$-dependent coefficient factor $1 /\left(6 c-r^{2}\right)^{2} r^{4}$, its minimum locates at $r_{0}=\sqrt{3 c}$ and another term $(\ln (r / e \Lambda))^{2}$ reaches its minimum at $r_{1}=e \Lambda$. Given that the parameter $\Lambda$ is tuned so that $r_{0}=r_{1}$, in the radial direction, the potential has a global minimum at $r=r_{0}$. Similar to helical phase inflation in the minimal supergravity, the potential (43) also shows helicoid structure, as presented in Figure 3.

The physical mass of $r$ in the region near the vacuum is

$$
m_{r}^{2}=\left.\frac{\left(2 c-r^{2} / 3\right)^{2}}{4 c} \frac{\partial^{2} V}{\partial r^{2}}\right|_{r=\sqrt{3 c}}=\left(4+\frac{1}{2 \theta^{2}}\right) H^{2}
$$

where $H$ is the Hubble constant during inflation. So the norm $r$ is strongly stabilized at $r_{0}$. If $r_{0}$ and $r_{1}$ are not equal but close to each other, $r$ will slightly shift during inflation but its mass remains above the Hubble scale and inflation is still approximately the single field inflation. 


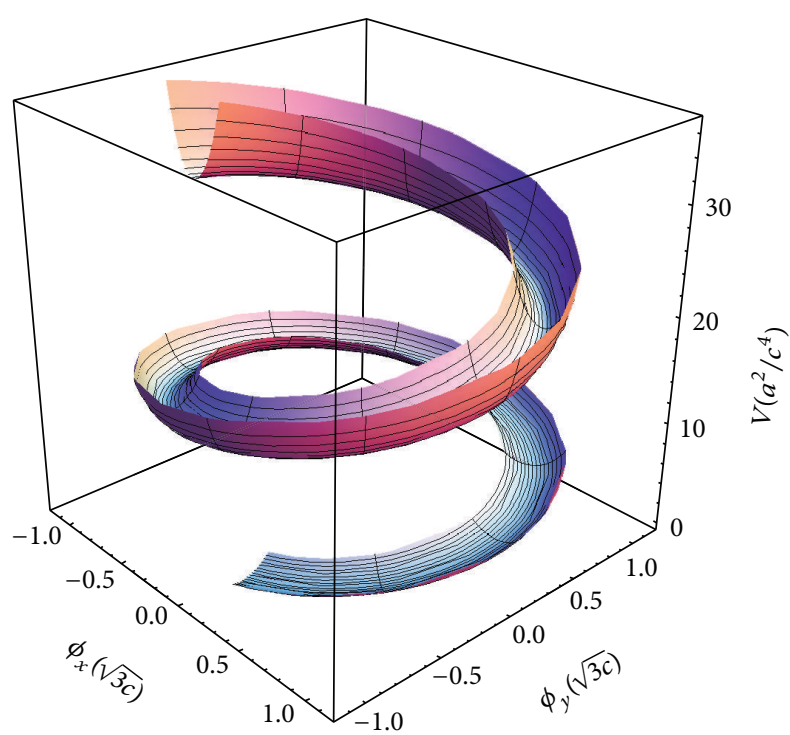

FIgURE 3: The helicoid structure of potential (43) scaled by $a^{2} / c^{4}$. In the graph, the parameter $\Lambda$ is tuned so that $r_{0}=r_{1}$. The complex field $\Phi$ has been rescaled by $\sqrt{3 c}$, and the scale of field norm at local valley is determined by the parameter $c$ instead of the Planck mass.

Instead of stabilizing $T$ independently with the inflation process, we can realize helical phase inflation from the noscale supergravity with dynamical $T$. There is a natural reason for such consideration. The phase monodromy requires matter fields transforming as rotations under $U(1)$ and also a modulus $T$ as a shift under $U(1)$. The Kähler potential of $T$ is shift invariant. Interestingly, for the no-scale supergravity with $S U(2,1) / S U(2) \times U(1)$ symmetry, as shown in (39), the Kähler potential $K$ is automatically endowed with the shift symmetry and global $U(1)$ symmetry:

$$
\begin{aligned}
& T \longrightarrow T+i C, \\
& \Phi \longrightarrow \Phi e^{i \theta} .
\end{aligned}
$$

Therefore, the no-scale Kähler potential fits with the phase monodromy in (16) and (22) initiatively.

The Kähler potentials of superfields $z \in X, Y, Z$ are of the minimal type $z \bar{z}$, while for $\Psi$, its Kähler potential can be the minimal type $\Psi \bar{\Psi}$, or the no-scale type $K=$ $-3 \ln (T+\bar{T}-(\Phi \bar{\Phi}+\Psi \bar{\Psi}) / 3)$, which extends the symmetry of Kähler manifold to $S U(3,1) / S U(3) \times U(1)$. In this scenario, the process to integrate out heavy fields is the same as before. Besides, the potential of phase proportional to $\left|W_{X}\right|^{2}$ is insensitive to the formula of Kähler potential due to $U(1)$ symmetry. The major difference appears in the field stabilization. The scalar potential from phase monodromy in (16) is

$$
\begin{aligned}
V= & e^{K}\left|W_{X}\right|^{2}=\frac{1}{\left(\ln r^{2} / \alpha+c-r^{2} / 3-\lambda^{2} r^{2} / 3\right)^{3}} \\
& \cdot \frac{1}{r^{2}}\left((\ln r-\ln \Lambda)^{2}+\theta^{2}\right) \approx \frac{1}{\left(c-r^{2} / 3\right)^{3}} \\
& \cdot \frac{1}{r^{2}}\left((\ln r-\ln \Lambda)^{2}+\theta^{2}\right),
\end{aligned}
$$

with $\alpha \gg 1$ and $\lambda \ll 1$. The field norm $r$ is stabilized by minimizing the coefficient $1 / r^{2}\left(c-r^{2} / 3\right)^{3}$ and the parameter $c$ from $\langle T\rangle$ determines the scale of $\langle r\rangle=\sqrt{3 c} / 2$. This is different from helical phase inflation in the minimal supergravity, which minimizes the norm based on the coefficient $e^{r^{2}}\left(1 / r^{2}\right)$, and then the scale of $\langle r\rangle$ is close to $M_{P}$, the unique energy scale of supergravity corrections. Similarly, combining the no-scale Kähler manifold with phase monodromy in (22), we can obtain natural inflation.

\section{UV-Sensitivity and Helical Phase Inflation}

For the slow-roll inflation, the Lyth bound [21] provides a relationship between the tensor-to-scalar ratio $\mathbf{r}$ and the field excursion $\Delta \phi$. Roughly it requires

$$
\frac{\Delta \phi}{M_{P}} \geqslant\left(\frac{\mathbf{r}}{0.01}\right)^{1 / 2} .
$$

To get large tensor-to-scalar ratio $\mathbf{r} \geqslant 0.01$, such as in chaotic inflation or natural inflation, the field excursion should be much larger than the Planck mass. The super-Planckian field excursion makes the description based on the effective field theory questionable. In the Wilsonian sense, the low energy field theory is an effective theory with higher order corrections introduced by the physics at the cut-off scale, like quantum gravity, and these terms are irrelevant in the effective field theory since they are suppressed by the cut-off energy scale. However, for the inflation process, the inflaton has super-Planckian field excursion, which is much larger than the cut-off scale. Thus, the higher order terms cannot be suppressed by the Planck mass and may affect the inflationary observations significantly. And then they may significantly affect inflation or even destroy the inflation process. For example, considering the corrections

$$
\Delta V=c_{i} V\left(\frac{\phi}{M_{P}}\right)^{i}+\cdots,
$$

to the original inflaton potential $V$, as long as $c_{i}$ are of the order $10^{-i}$, in the initial stage of inflation $\phi \sim O(10) M_{P}$, the higher order terms can be as large as the original potential $V$. So for large field inflation, it is sensitive to the physics at the cut-off scale and the predictions of inflation just based on effective field theory are questionable.

In consideration of the UV sensitivity of large field inflation, a possible choice is to realize inflation in UVcompleted theory, like string theory (for a review, see [22]). To realize inflation in string theory, there are a lot of problems to solve besides inflation, such as the moduli stabilization, Minkowski/de Sitter vacua, and effects of $\alpha^{\prime}$ - and string loopcorrections. Alternatively, in the bottom-up approach, one may avoid the higher order corrections by introducing an extra shift symmetry in the theory. The shift symmetry is technically natural and safe under quantum loop-corrections. However, the global symmetry can be broken by the quantum gravity effect. So it is still questionable whether the shift symmetry can safely evade the higher corrections like in (48). 
The UV-completion problem is dodged in helical phase inflation. Since the super-Planckian field excursion is the phase of a complex field and the phase component is not directly involved in the gravity interaction, there are no dangerous high-order corrections like in (48) for the phase potential. The inflaton evolves along the helical trajectory and does not relate to the physics in the region above the Planck scale. For helical phase inflation in the minimal supergravity, the norm of field is stabilized at the marginal point of the Planck scale, where the supergravity correction on the scalar potential gets important based on which the norm of complex field can be strongly stabilized. The extra corrections are likely to appear in the Kähler potential; however, they can only slightly affect the field stabilization while the phase inflation is protected by the global $U(1)$ symmetry in the Kähler potential; in consequence, helical phase inflation is not sensitive to these corrections at all. For the helical phase inflation in no-scale supergravity, the norm of complex field is stabilized at the scale of the modulus $\langle T\rangle$ instead of the Planck scale; one can simply adjust the scale of $\langle T\rangle$ to keep the model away from super-Planckian region.

Helical phase inflation is free from the UV-sensitivity problem, and it is just a typical physical process at the GUT scale with special superpotential that admits phase monodromy. So it provides an inflationary model that can be reliably studied just in supersymmetric field theory.

\section{Discussions and Conclusion}

In this work, we have studied the details of helical phase inflation from several aspects. Helical phase inflation is realized in supergravity with global $U(1)$ symmetry. $U(1)$ symmetry is built in the Kähler potential so that helical phase inflation can be realized by the ordinary Kähler potentials, such as the minimal or no-scale types. Helical phase inflation directly leads to the phase monodromy in the superpotential, which is singular and an effective field theory arising from integrating out heavy fields. The phase monodromy originates from $U(1)$ rotation of the superpotential at higher scale. Generically, the superpotential can be separated into two parts $W=W_{I}+W_{S}$, where $W_{S}$ admits the global $U(1)$ symmetry while $W_{I}$ breaks it explicitly at scale much lower than $W_{S}$. Under $U(1)$ rotation, the inflation term $W_{I}$ is slightly changed, which realizes the phase monodromy in the effective theory and introduces a flat potential along the direction of phase rotation. By breaking the global $U(1)$ symmetry in different ways, we may get different kinds of inflation such as quadratic inflation, natural inflation, or the other types of inflation that are not presented in this work.

An amazing fact of helical phase inflation is that it deeply relates to several interesting points of inflation and naturally combines them in a rather simple potential with helicoid structure. The features of helical phase inflation can be summarized as follows:

(i) The global $U(1)$ symmetry is built in the Kähler potential, so helical phase inflation provides a natural solution to $\eta$ problem. (ii) The phase excursion requires phase monodromy in the superpotential. So helical phase inflation provides, in the mathematical sense, a new type of monodromy in supersymmetric field theory.

(iii) The singularity in the superpotential, together with the supergravity scalar potential, provides strong field stabilization which is consistent with phase inflation.

(iv) The super-Planckian field excursion is realized by the phase of a complex field instead of any other "physical" fields that directly couple with gravity. So there are no polynomial higher order corrections for the phase and thus inflation is not sensitive to the quantum gravity corrections.

To summarize, helical phase inflation introduces a new type of inflation that can be effectively described by supersymmetric field theory at the GUT scale. Generically, the super-Planckian field excursion makes the inflationary predictions based on effective field theory questionable, since the higher order corrections from quantum gravity are likely to affect the inflation process significantly. One of the solutions is to realize inflation in a UV-completed theory, like string theory; nevertheless, there are many difficult issues in string theory to resolve before realizing inflation completely. Helical phase inflation is another simple solution to the UVsensitivity problem. It is based on the supersymmetric field theory and the physics are clear and much easier to control. Furthermore, helical phase inflation makes the unification of inflation theory with GUT more natural, since both of them are triggered at the scale of $10^{16} \mathrm{GeV}$ and can be effectively studied based on supersymmetric field theory.

Besides, we have shown that helical phase inflation also relates to several interesting developments in inflation theory. It can be easily modified for natural inflation and realize the phase-axion alignment indirectly, which is similar to the axion-axion alignment mechanism for super-Planckian axion decay constant [34]. The phase-axion alignment is not shown in the final supergravity model which exhibits explicit phase monodromy only. However, the phase-axion alignment is hidden in the process when the heavy fields are integrating out. For $\eta$ problem in the supergravity inflation, there is another well-known solution, the KYY model with shift symmetry. We showed that through field redefinition helical phase inflation given by (4) and (5) reduces to the KYY model, where the higher order corrections in the Kähler potential have no effect on inflation process. However, there is no inverse transformation from the KYY model to helical phase inflation since no well-defined field redefinition can introduce the pole and phase singularity needed for phase monodromy. Helical phase inflation can be realized in noscale supergravity. The no-scale Kähler potential automatically provides the symmetry needed by phase monodromy. In the no-scale supergravity, the norm of complex field is stabilized at the scale of the modulus.

Our inflation models are constructed within the supergravity theory with global $U(1)$ symmetry broken explicitly by the subleading order superpotential term. So it is just typical GUT scale physics and indicates that a UV-completed 
framework seems to be not prerequisite to effectively describe such inflation process.

\section{Conflict of Interests}

The authors declare that there is no conflict of interests regarding the publication of this paper.

\section{Acknowledgments}

The work of DVN was supported in part by the DOE Grant DE-FG03-95-ER-40917. The work of Tianjun Li is supported in part by the Natural Science Foundation of China under Grants nos. 10821504, 11075194, 11135003, 11275246, and 11475238 and by the National Basic Research Program of China (973 Program) under Grant no. 2010CB833000.

\section{References}

[1] A. H. Guth, "Inflationary universe: a possible solution to the horizon and flatness problems," Physical Review D, vol. 23, no. 2, pp. 347-356, 1981.

[2] A. D. Linde, "Coleman-Weinberg theory and the new inflationary universe scenario," Physics Letters B, vol. 114, no. 6, pp. 431435, 1982.

[3] A. Albrecht and P. J. Steinhardt, "Cosmology for grand unified theories with radiatively induced symmetry breaking," Physical Review Letters, vol. 48, no. 17, pp. 1220-1223, 1982.

[4] P. A. R. Ade, N. Aghanim, C. Armitage-Caplan et al., "Planck 2013 results. XXII. Constraints on inflation," Astronomy \& Astrophysics, vol. 571, article A22, 42 pages, 2014.

[5] P. A. R. Ade, R. W. Aikin, D. Barkats et al., "Detection of Bmode polarization at degree angular scales by BICEP2," Physical Review Letters, vol. 112, no. 24, Article ID 241101, 2014.

[6] M. Kawasaki, M. Yamaguchi, and T. Yanagida, "Natural chaotic inflation in supergravity," Physical Review Letters, vol. 85, no. 17, pp. 3572-3575, 2000.

[7] T. Li, Z. Li, and D. V. Nanopoulos, "Supergravity inflation with broken shift symmetry and large tensor-to-scalar ratio," Journal of Cosmology and Astroparticle Physics, vol. 2014, no. 2, article 028, 2014.

[8] K. Harigaya and T. T. Yanagida, "Discovery of large scale tensor mode and chaotic inflation in supergravity," Physics Letters $B$, vol. 734, pp. 13-16, 2014.

[9] J. Ellis, D. V. Nanopoulos, and K. A. Olive, "No-scale supergravity realization of the starobinsky model of inflation," Physical Review Letters, vol. 111, no. 11, Article ID 111301, 2013.

[10] T. Li, Z. Li, and D. V. Nanopoulos, "No-scale ripple inflation revisited," Journal of Cosmology and Astroparticle Physics, vol. 04, article 018, 2014.

[11] C. Pallis, "Induced-gravity inflation in no-scale supergravity and beyond," Journal of Cosmology and Astroparticle Physics, vol. 2014, no. 8, article 057, 2014.

[12] S. Ferrara, A. Kehagias, and A. Riotto, "The imaginary Starobinsky model," Fortschritte der Physik, vol. 62, no. 7, pp. 573-583, 2014.

[13] J. Ellis, M. A. G. Garca, D. V. Nanopoulos, and K. A. Olive, "Resurrecting quadratic inflation in no-scale supergravity in light of BICEP2," Journal of Cosmology and Astroparticle Physics, vol. 2014, no. 5, article 037, 2014.
[14] T. Li, Z. Li, and D. V. Nanopoulos, "Chaotic inflation in noscale supergravity with string inspired moduli stabilization," The European Physical Journal C, vol. 75, no. 2, article 55, 2015.

[15] S. Ferrara, A. Kehagias, and A. Riotto, "The imaginary Starobinsky model and higher curvature corrections," Fortschritte der Physik, vol. 63, no. 1, pp. 2-11, 2015.

[16] S. V. Ketov and T. Terada, "Inflation in supergravity with a single chiral superfield," Physics Letters B, vol. 736, pp. 272-277, 2014.

[17] C. Kounnas, D. Lüst, and N. Toumbas, "R2 inflation from scale invariant supergravity and anomaly free superstrings with fluxes," Fortschritte der Physik, vol. 63, no. 1, pp. 12-35, 2015.

[18] G. A. Diamandis, B. C. Georgalas, K. Kaskavelis, P. Kouroumalou, A. B. Lahanas, and G. Pavlopoulos, "Inflation in $\mathrm{R}^{2}$ supergravity with non-minimal superpotentials," Physics Letters B, vol. 744, pp. 74-81, 2015.

[19] C. Pallis and Q. Shafi, "Gravity waves from non-minimal quadratic inflation," Journal of Cosmology and Astroparticle Physics, vol. 2015, no. 3, article 023, 2015.

[20] T. Li, Z. Li, and D. V. Nanopoulos, "Helical phase inflation," Physical Review D, vol. 91, no. 6, Article ID 061303, 2015.

[21] D. H. Lyth, "What would we learn by detecting a gravitational wave signal in the cosmic microwave background anisotropy?" Physical Review Letters, vol. 78, no. 10, article 1861, 1997.

[22] D. Baumann and L. McAllister, "Inflation and stringtheory," http://arxiv.org/abs/1404.2601.

[23] F. Marchesano, G. Shiu, and A. M. Uranga, "F-term axion monodromy inflation," Journal of High Energy Physics, vol. 2014, no. 9, article 184, 2014.

[24] C. Long, L. McAllister, and P. McGuirk, "Aligned natural inflation in string theory," Physical Review D, vol. 90, no. 2, Article ID 023501, 2014.

[25] X. Gao, T. Li, and P. Shukla, "Combining universal and odd RR axions for aligned natural inflation," Journal of Cosmology and Astroparticle Physics, vol. 2014, no. 10, article 048, 2014.

[26] I. Ben-Dayan, F. G. Pedro, and A. Westphal, “Towards natural inflation in string theory," Physical Review D, vol. 92, no. 2, Article ID 023515, 2015.

[27] R. Blumenhagen, D. Herschmann, and E. Plauschinn, "The challenge of realizing F-term axion monodromy inflation in string theory," Journal of High Energy Physics, vol. 2015, article 7, 2015.

[28] H. Abe, T. Kobayashi, and H. Otsuka, "Towards natural inflation from weakly coupled heterotic string theory," Progress of Theoretical and Experimental Physics, Article ID 063E02, 2015.

[29] T. Ali, S. S. Haque, and V. Jejjala, "Natural inflation from near alignment in heterotic string theory," Physical Review D, vol. 91, no. 8, Article ID 083516, 9 pages, 2015.

[30] R. Flauger, L. McAllister, E. Silverstein, and A. Westphal, "Drifting oscillations in axionmonodromy," http://arxiv.org/abs/1412 .1814 .

[31] K. Freese, J. A. Frieman, and A. V. Olinto, "Natural inflation with pseudo nambu-goldstone bosons," Physical Review Letters, vol. 65, no. 26, pp. 3233-3236, 1990.

[32] F. C. Adams, J. R. Bond, K. Freese, J. A. Frieman, and A. V. Olinto, "Natural inflation: particle physics models, powerlaw spectra for large-scale structure, and constraints from the cosmic background explorer," Physical Review D, vol. 47, no. 2, pp. 426-455, 1993.

[33] G. Germán, A. Mazumdar, and A. Pérez-Lorenzana, "Angular inflation from supergravity," Modern Physics Letters A, vol. 17, no. 25, pp. 1627-1634, 2002. 
[34] J. E. Kim, H. P. Nilles, and M. Peloso, "Completing natural inflation," Journal of Cosmology and Astroparticle Physics, vol. 0501, article 005, 2005.

[35] D. Baumann and D. Green, "Inflating with baryons," Journal of High Energy Physics, vol. 2011, no. 4, article 071, 2011.

[36] J. McDonald, "Sub-Planckian two-field inflation consistent with the Lyth bound," Journal of Cosmology and Astroparticle Physics, vol. 2014, no. 9, article 027, 2014.

[37] J. McDonald, "A minimal sub-Planckian axion inflation model with large tensor-to-scalar ratio," Journal of Cosmology and Astroparticle Physics, vol. 2015, no. 1, article 018, 2015.

[38] C. D. Carone, J. Erlich, A. Sensharma, and Z. Wang, "Two-field axion-monodromy hybrid inflation model: Dante's Waterfall," Physical Review D, vol. 91, no. 4, Article ID 043512, 8 pages, 2015.

[39] G. Barenboim and W.-I. Park, "Spiral Inflation," Physics Letters B, vol. 741, pp. 252-255, 2015.

[40] T. Li, Z. Li, and D. V. Nanopoulos, "Aligned natural inflation and moduli stabilization from anomalous $U(1)$ gauge symmetries," Journal of High Energy Physics, vol. 2014, no. 11, article 012, 2014.

[41] T. Li, Z. Li, and D. V. Nanopoulos, "Natural inflation with natural trans-planckian axion decay constant from anomalous $\mathrm{U}(1)_{X}$," Journal of High Energy Physics, vol. 2014, no. 7, article 052, 2014.

[42] E. Silverstein and A. Westphal, "Monodromy in the CMB: gravity waves and string inflation," Physical Review D, vol. 78, no. 10, Article ID 106003, 21 pages, 2008.

[43] L. McAllister, E. Silverstein, and A. Westphal, "Gravity waves and linear inflation from axion monodromy," Physical Review $D$, vol. 82, no. 4, Article ID 046003, 2010.

[44] K. Choi, H. Kim, and S. Yun, "Natural inflation with multiple sub-Planckian axions," Physical Review D, vol. 90, no. 2, Article ID 023545, 2014.

[45] S.-H. H. Tye and S. S. C. Wong, "Helical inflation and cosmicstrings," http://arxiv.org/abs/1404.6988.

[46] R. Kappl, S. Krippendorf, and H. P. Nilles, "Aligned natural inflation: monodromies of two axions," Physics Letters B, vol. 737, pp. 124-128, 2014.

[47] G. 'tHooft, "Naturalness, chiral symmetry, and spontaneous chiral symmetry breaking," in Recent Developments in Gauge Theories, G. 'tHooft, C. Itzykson, A. Jaffe et al., Eds., vol. 59 of NATO Advanced Study Institutes Series, pp. 135-157, Plenum Press, New York, NY, USA, 1980.

[48] R. Blumenhagen, M. Cvetic, S. Kachru, and T. Weigand, "Dbrane instantons in type II string theory," Annual Review of Nuclear and Particle Science, vol. 59, pp. 269-296, 2009.

[49] K. Harigaya and M. Ibe, "Simple realization of inflaton potential on a Riemann surface," Physics Letters B, vol. 738, pp. 301-304, 2014.

[50] R. Kallosh, A. Linde, and B. Vercnocke, "Natural inflation in supergravity and beyond," Physical Review D, vol. 90, Article ID 041303(R), 2014. 

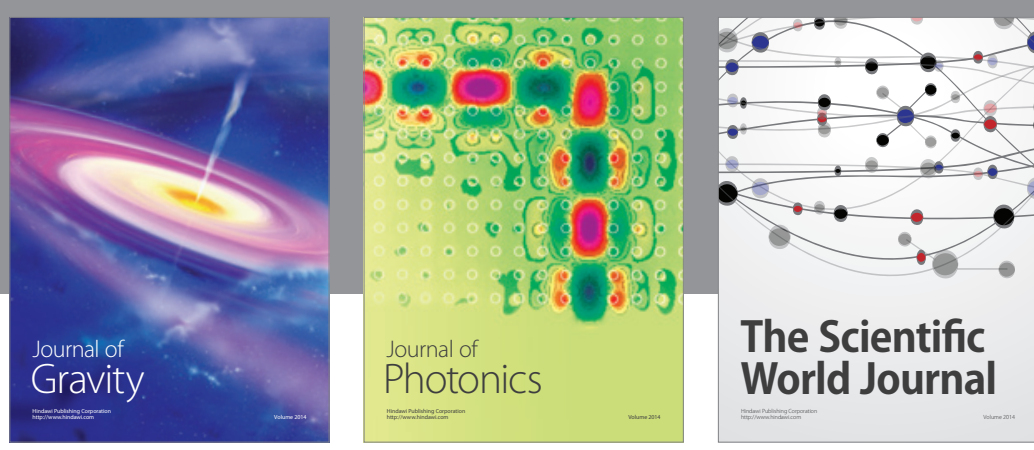

The Scientific World Journal
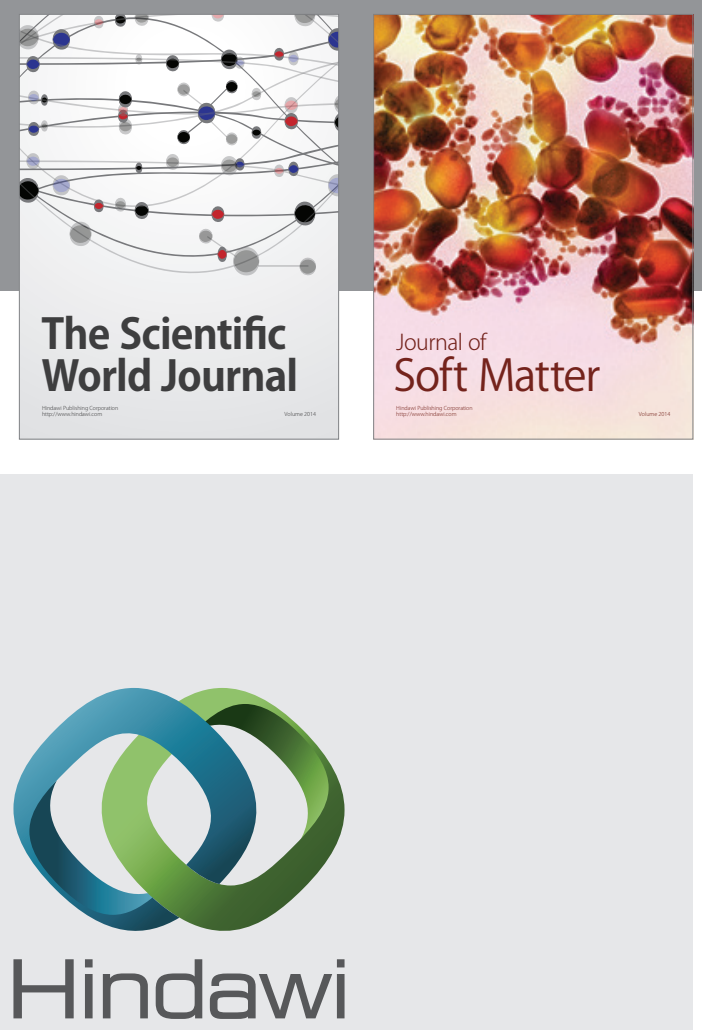

Submit your manuscripts at

http://www.hindawi.com

nternational Journal of

Statistical Mechanics
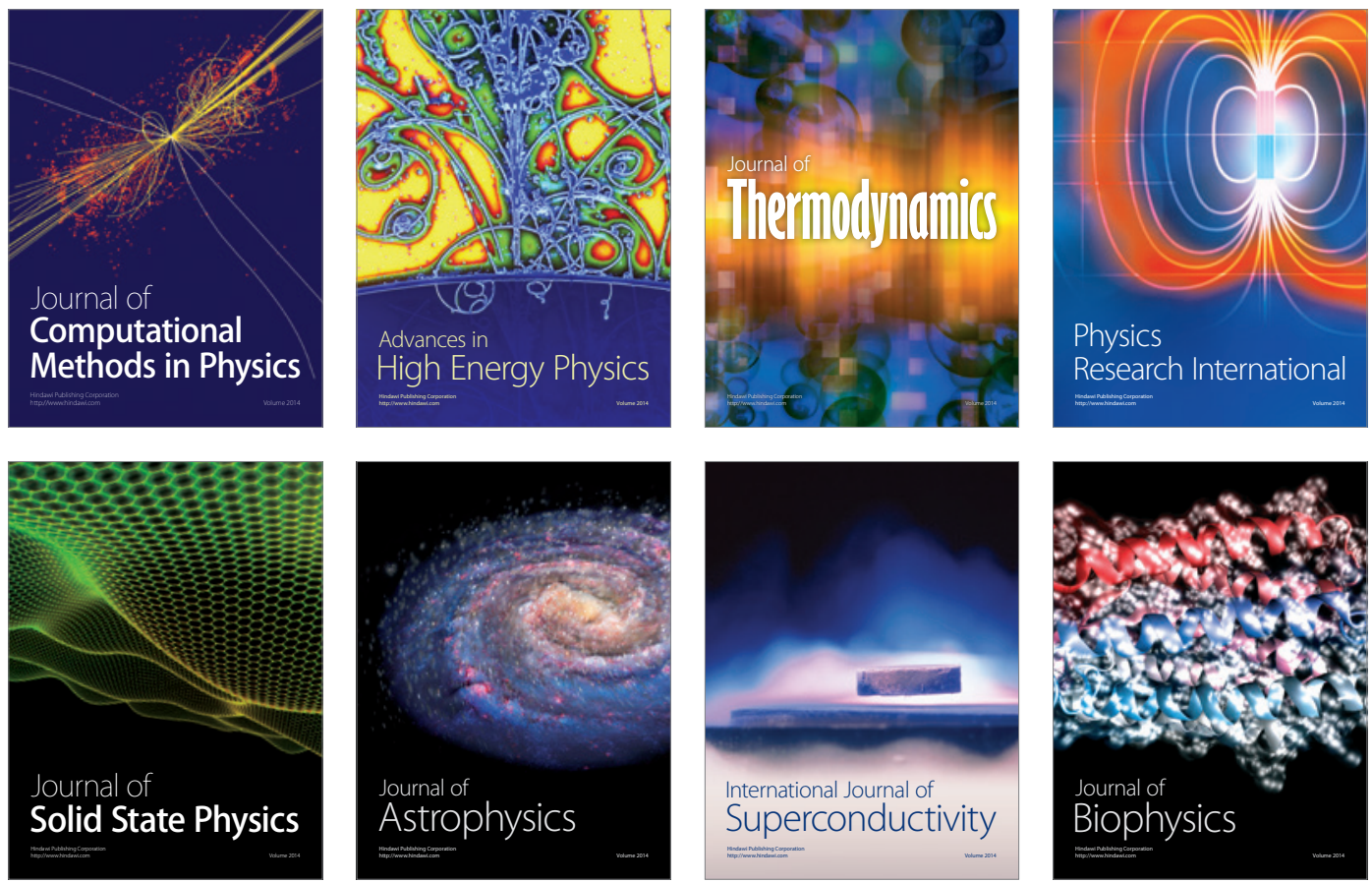
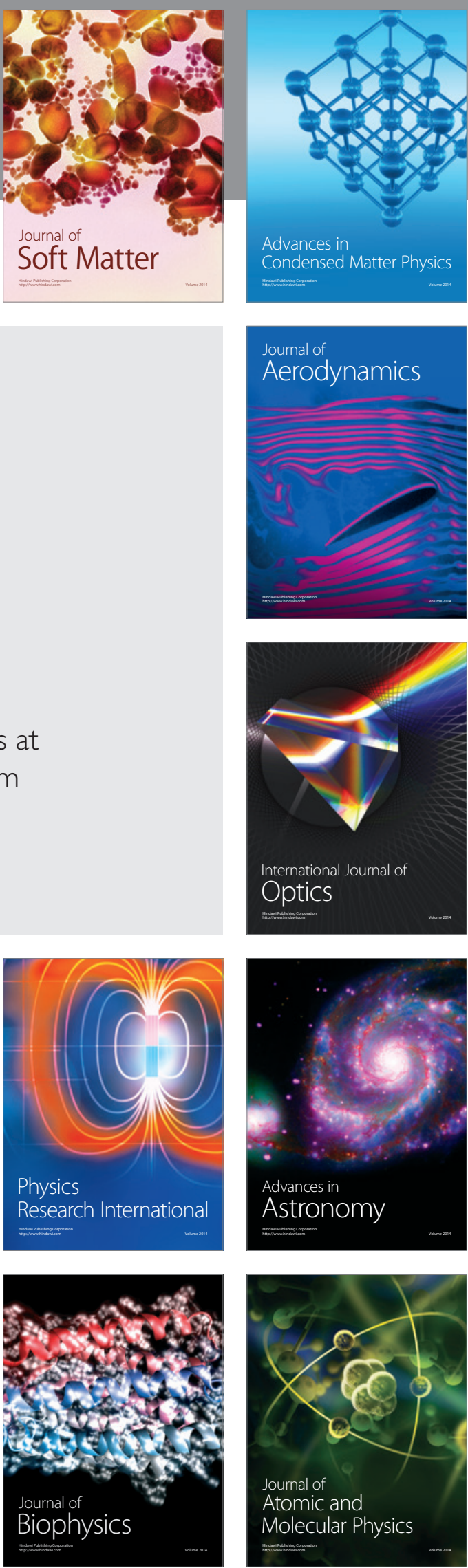